\title{
Comparison of specific IgE detection by immunoblotting and fluorescence enzyme assay with in vivo skin prick test
}

\author{
Jongkonnee Wongpiyabovorn, ${ }^{1}$ Narissara Suratannon, ${ }^{2}$ Sadudee Boonmee, ${ }^{2}$ Pantipa Chatchatee ${ }^{2}$
}

\begin{abstract}
Background: Diagnostic tools to identify allergens that cause allergic symptom is important part in the care of allergic patients. Detection of causative allergen can be performed by in vivo skin prick test (SPT) or in vitro tests for detection serum specific immunoglobulin E (sIgE). The common methods used are fluorescent enzyme assay and immunoblotting assay.
\end{abstract}

Objective: We aim to evaluate performance of the two sIgE determination systems, immunoblotting assay (Euroline) and fluorescent enzyme assay (ImmunoCAP) in comparison with SPT.

Methods: Two hundred and two participants with allergic diseases were enrolled. Sensitization to common allergens was identified using skin prick test and serum specific IgE assays with Euroline and ImmunoCAP. Both systems provide the result in the same unit and the same cut-off value $(0.35 \mathrm{kUA} / \mathrm{L})$. The specific IgE levels of 4 aeroallergens, 6 food allergens and 3 food allergen components were analyzed to evaluate the performance of both sIgE assays with SPT.

Results: When compared with the result of SPT, ImmunoCAP has 63.9-93.2\% agreement and Euroline has 68.4-86.2\% agreement for allergen detection. Both sIgE assays have significant correlation in measuring sIgE of almost all allergens $(\mathrm{r}=0.626-0.901, \mathrm{p}<0.001)$ except for dog. For food allergen components, both sIgE tests have outstanding correlation and agreement $(\mathrm{r}=0.816-0.952, \mathrm{p}<0.001$; agreement $=87.0-92.9 \%$, respectively $)$. The receiver-operating characteristic curve analysis indicated slight discrepancy of both sIgE assays.

Conclusions: Both sIgE determination systems demonstrate fair to good performance when compared to SPT depending on type of allergens. The two sIgE determination systems had favorable correlation and agreement.

Keywords: Fluorescence enzyme assay, Immunoblotting assay, Specific IgE assay, Skin prick test, allergen.

\section{From:}

${ }^{1}$ Center of Excellence in Immunology and Immune Mediated Diseases, Department of Microbiology, Faculty of Medicine, Chulalongkorn University, Bangkok, Thailand

${ }^{2}$ Division of Allergy and Immunology, Department of Pediatrics,

Faculty of Medicine, Chulalongkorn University, Bangkok, Thailand

\section{Corresponding author:}

Pantipa Chatchatee

Division of Allergy and Immunology, Department of Pediatrics

Faculty of Medicine, Chulalongkorn University

Rama 4 Road Bangkok 10330 Thailand

E-mail: pantipa1111@gmail.com

\section{Introduction}

Allergic diseases have been increasing around the world. To identify causative allergens is the important part of diagnosis and treatment. Detection of causative allergen by skin prick test (SPT) is commonly used because of the high sensitivity, ${ }^{1,2}$ rapidity and inexpensiveness. Allergen-specific immunoglobulin E (sIgE) blood assay offers the alternative tool to identify the causative allergen. The advantages of sIgE blood assay are the variety of assays including quantitative or semi-quantitative system, lack of medication/ skin condition interference and no risk of severe allergic reaction occurred during the assay.
In general, good agreement has been identified between skin test and sIgE blood assay for the most potent aeroallergens including trees, grasses, weeds, pets (eg, cat and dog), and dust mite allergens. ${ }^{1,3-6}$ Nevertheless, some studies revealed the discrepancy between SPT and sIgE blood assay. ${ }^{4,6,7}$ Variability in skin test and sIgE results may due to several factors such as patients' factors, method of SPT, quality and stability of the allergen extracts, the biological reagents used in the laboratory assay and the methods of laboratory assay. ${ }^{1}$

Recently, various commercial analytical system for sIgE 
detection have been developed for example radioimmunoassay, chemiluminescence, enzyme-immunoassay, fluorescence/ enzyme-immunoassay and immunoblotting assay. Only few sIgE determination methods have been approved by United State Food and Drug Administration (US FDA) such as ImmunoCAP (Phadia, Uppsala, Sweden), Turbo RAST (Agilent Technologies Co, Santa Clara, California) and Immulite (Siemens Medical Solutions Diagnostics, Tarrytown, New York). Despite these systems provided the result in the same unit, the result is not always equivalent. ${ }^{3,8-10}$

Euroline (Euroimmun, Medizinische Labordiagnostika, AG, Germany), an IgE determination system using immunoblotting assay has some advantages such as the requirement of only small amount of sera, no automate machine requirement, minimal hands-on time, appropriate for screening multiple allergen and cost-saving. While ImmunoCAP (Phadia, Uppsala, Sweden), a fluorescence/enzyme-immunoassay, has some advantages such as high through-put with a lot of number of sample load per day but it has some disadvantage such as automate machine requirement and expensive. Nowadays, there has been no study to evaluate the performance of sIgE detection systems using immunoblotting assay compared to the widely-used in vivo SPT. Our study aimed to evaluate the performance of an immunoblot (Euroline) and a fluorescence/enzyme-immunoassay (ImmunoCAP) for the detection and quantitation of sIgE in comparison with in vivo SPT.

\section{Methods \\ Material and Methods}

The study has been carried out at out-patient clinic, King Chulalongkorn Hostpital during June 2012 to October 2015. Two hundred and two subjects with allergic diseases: asthma, allergic rhinitis, atopic dermatitis, food allergy and anaphylaxis, age from 1 month to 60 years, were enrolled. Subjects with uncontrolled cardiovascular and respiratory diseases other than allergic asthma, pregnant or lactating women, or patient who received allergen immunotherapy, systemic immunosuppressive drugs, beta-blocking agents were excluded. All subjects were free from anti-histamine for at least 7 days prior to the procedure. The study was approved by Ethics Committee. Informed consent was obtained.

\section{Skin prick test method}

SPT was performed by the prick technique using a metal lancet (Vitrex ${ }^{\oplus}$ steel, Vitrex Medical A/S, Herlev, Denmark) on the volar side of the forearm of the subjects. All extracts were supplied by ALK-Abello (Hørsholm, Denmark). Histamine Dihydrochloride in $50 \%$ glycerin $(1 \mathrm{mg} / \mathrm{mL})$ and $50 \%$ glycerosaline were served as the positive and negative control accordingly. Commercially available allergens were used as the following; house dust mites extract (Dermatophagoides pteronyssinus (Der p), Dermatophagoides faerinae (Der f)); cat hair extract; dog epithelial extract; shrimp extract; crab extract; egg white extract; egg yolk extract; and wheat extract. Skin prick test was read at fifteen minutes for allergens and ten minutes for positive control. A positive skin response was defined as the presence of a wheal with a mean wheal diameter of at least 3 millimeters $(\mathrm{mm})$ greater than that elicited by the negative control accompanied by erythema. The mean of the largest and midpoint orthogonal diameters was designated as the mean wheal diameter (MWD).

\section{Serum specific IgE assays}

Serum samples were collected from the participants at the same visit as SPT, then divided into 2 aliquots and store at $-20^{\circ} \mathrm{C}$ until use. The level of sIgE was quantified using fluorescence enzyme immunoassay (PharmaciaCAP, Pharmacia) and immunoblotting assay (Euroline, Medizinische Labordiagnostika AG) according to the manufacturer's instructions. SIgE for Der p, Der f, cat, dog, egg white, egg yolk, cow's milk, wheat, shrimp and food allergen components (gluten/omega-5 gliadin, ovalbumin, ovomucoid) were measured. Level of specific $\operatorname{IgE} \geq$ $0.35 \mathrm{kUA} / \mathrm{L}$ (Class 1) was considered to be positive result for both assays.

\section{Statistical analysis}

The sensitivity, specificity, positive predictive value (PPV), negative predictive value (NPV) and accuracy of Euroline and ImmunoCAP were analyzed according to SPT for each allergen. The correlation of sIgE level and SPT as well as between Euroline and ImmunoCAP were analyzed using Spearman's rho correlation. A receiver-operating characteristic (ROC) curve analysis was performed to verify the performance of Euroline and ImmunoCAP with SPT. Agreement between sIgE and SPT was assessed by kappa statistics.

\section{Results \\ Study population}

There were 202 participants, age from 1 month to 60 years (mean $\pm \mathrm{SD}=10.57 \pm 14.13$ ), in the study. Fifty-seven were male and 145 were female. One hundred and fifty nine participants age $\leq 17$ years. The underlying allergic diseases were asthma $(n=9,4.46 \%)$, allergic rhinitis and allergic rhino -conjunctivitis $(\mathrm{n}=58,28.71 \%)$, atopic dermatitis $(\mathrm{n}=28$, $13.86 \%)$, food allergy $(n=86,42.57 \%)$, urticaria $(n=18,8.91 \%)$ and anaphylaxis $(n=3,1.49 \%)$.

\section{Performance of Immunoblotting assay (Euroline) and Fluores- cence enzyme assay (ImmunoCAP) compared with skin prick test}

The performance of two vitro sIgE assays for each allergen was compared to the skin prick test as shown in Table 1. Using the SPT cut-off mean wheal diameter of at least 3 millimeters and sIgE cut-off value ( $\geq 0.35 \mathrm{kUA} / \mathrm{L})$ (class I), overall agreement of ImmunoCAP and SPT were 63.9-93.2\% while the agreement of Euroline and SPT was $68.4-86.2 \%$. When compared to SPT, both Euroline and ImmunoCAP displayed high sensitivity, specificity, PPV and agreement for Der $p$, Der $\mathrm{f}$ and crab sIgE detection. However, Euroline had lower sensitivity but higher specificity than ImmunoCAP for cat, dog and wheat sIgE detection (sensitivity 48.0\%, 33.3\%, $31.4 \%$ and specificity $96.6 \%, 96.7 \%, 90.0 \%$, respectively). 
Table 1. Performance of ImmunoCAP and Euroline compared with SPT for each allergen

a) The analysis at SPT cut-off $3 \mathrm{~mm}$ and class I sIgE

\begin{tabular}{|c|c|c|c|c|c|c|c|c|}
\hline Allergen & System (n) & $\begin{array}{c}\% \text { of positive } \\
\text { test }\end{array}$ & $\begin{array}{c}\text { Sensitivity } \\
(\%)\end{array}$ & $\begin{array}{c}\text { Specificity } \\
(\%)\end{array}$ & $\begin{array}{l}\text { PPV } \\
(\%)\end{array}$ & $\begin{array}{c}\text { NPV } \\
(\%)\end{array}$ & $\begin{array}{c}\text { Agreement } \\
(\%)\end{array}$ & $\begin{array}{c}\text { Kappa } \\
(95 \% \text { CI. })\end{array}$ \\
\hline \multirow[t]{2}{*}{ Der p } & ImmunoCAP (88) & 78.41 & 97.1 & 78.9 & 94.4 & 88.2 & 93.2 & $0.791(0.631-0.951)$ \\
\hline & Euroline (161) & 56.52 & 82.4 & 88.6 & 90.4 & 79.5 & 85.1 & $0.701(0.591-0.811)$ \\
\hline \multirow[t]{2}{*}{ Der $\mathbf{f}$} & ImmunoCAP (64) & 68.75 & 97.7 & 80.0 & 91.5 & 94.1 & 92.2 & $0.81(0.652-0.969)$ \\
\hline & Euroline (87) & 71.26 & 85.5 & 88.0 & 94.6 & 71.0 & 86.2 & $0.686(0.524-0.848)$ \\
\hline \multirow[t]{2}{*}{ Cat } & ImmunoCAP (26) & 57.69 & 66.7 & 72.7 & 76.9 & 61.5 & 69.2 & $0.385(0.034-0.735)$ \\
\hline & Euroline (112) & 22.32 & 48.0 & 96.6 & 80.0 & 86.6 & 85.7 & $0.52(0.319-0.72)$ \\
\hline \multirow[t]{2}{*}{ Dog } & ImmunoCAP (36) & 16.67 & 100.0 & 66.7 & 37.5 & 100.0 & 72.2 & $0.4(0.147-0.653)$ \\
\hline & Euroline (100) & 9.0 & 33.3 & 96.7 & 50.0 & 93.6 & 91.0 & $0.353(0.026-0.681)$ \\
\hline \multirow[t]{2}{*}{ Egg white } & ImmunoCAP (76) & 61.84 & 83.0 & 55.2 & 75.0 & 66.7 & 72.4 & $0.395(0.181-0.608)$ \\
\hline & Euroline (106) & 54.72 & 81.0 & 75.0 & 79.7 & 76.6 & 78.3 & $0.561(0.403-0.72)$ \\
\hline \multirow[t]{2}{*}{ Egg yolk } & ImmunoCAP (31) & 74.19 & 52.2 & 100.0 & 100.0 & 42.1 & 64.5 & $0.36(0.127-0.594)$ \\
\hline & Euroline (61) & 59.02 & 61.1 & 92.0 & 91.7 & 62.2 & 73.8 & $0.495(0.297-0.692)$ \\
\hline \multirow[t]{2}{*}{ Cow's milk } & ImmunoCAP (78) & 39.74 & 77.4 & 68.1 & 61.5 & 82.1 & 71.8 & $0.436(0.24-0.631)$ \\
\hline & Euroline (113) & 35.40 & 52.5 & 83.6 & 63.6 & 76.3 & 72.6 & $0.375(0.196-0.555)$ \\
\hline \multirow[t]{2}{*}{ Wheat } & ImmunoCAP (72) & 44.44 & 59.4 & 67.5 & 59.4 & 67.5 & 63.9 & $0.269(0.045-0.492)$ \\
\hline & Euroline (95) & 36.84 & 31.4 & 90.0 & 64.7 & 69.2 & 68.4 & $0.24(0.052-0.428)$ \\
\hline \multirow[t]{2}{*}{ Shrimp } & ImmunoCAP (72) & 65.28 & 87.2 & 64.0 & 82.0 & 72.7 & 79.2 & $0.527(0.319-0.736)$ \\
\hline & Euroline (154) & 35.06 & 63.0 & 94.0 & 85.0 & 82.5 & 83.1 & $0.606(0.472-0.739)$ \\
\hline \multirow[t]{2}{*}{ Crab } & ImmunoCAP (17) & 47.06 & 87.5 & 66.7 & 70.0 & 85.7 & 76.5 & $0.534(0.146-0.923)$ \\
\hline & Euroline (19) & 47.37 & 88.9 & 70.0 & 72.7 & 87.5 & 78.9 & $0.582(0.227-0.938)$ \\
\hline
\end{tabular}

b) The analysis at SPT cut-off $5 \mathrm{~mm}$ and class III sIgE

\begin{tabular}{|c|c|c|c|c|c|c|c|c|}
\hline Allergen & System (n) & $\begin{array}{c}\% \text { of positive } \\
\text { test }\end{array}$ & $\begin{array}{c}\text { Sensitivity } \\
(\%)\end{array}$ & $\begin{array}{c}\text { Specificity } \\
(\%)\end{array}$ & $\begin{array}{l}\text { PPV } \\
(\%)\end{array}$ & $\begin{array}{c}\text { NPV } \\
(\%)\end{array}$ & $\begin{array}{c}\text { Agreement } \\
(\%)\end{array}$ & $\begin{array}{c}\text { Kappa } \\
(95 \% \text { CI.) }\end{array}$ \\
\hline \multirow[t]{2}{*}{$\operatorname{Der} \mathbf{p}$} & ImmunoCAP (88) & 71.59 & 85.7 & 96.0 & 98.2 & 72.7 & 88.6 & $0.75(0.6-0.89)$ \\
\hline & Euroline (161) & 50.31 & 74.1 & 97.5 & 96.8 & 78.8 & 85.7 & $0.72(0.61-0.82)$ \\
\hline \multirow[t]{2}{*}{ Der $\mathbf{f}$} & ImmunoCAP (64) & 59.38 & 100 & 88.5 & 92.7 & 100 & 95.3 & $0.9(0.79-1)$ \\
\hline & Euroline (87) & 60.92 & 84.9 & 91.2 & 93.8 & 79.5 & 87.4 & $0.74(0.6-0.88)$ \\
\hline \multirow[t]{2}{*}{ Cat } & ImmunoCAP (26) & 34.62 & 22 & 100 & 100 & 70.8 & 73.1 & $0.27(-0.04-(-0.59))$ \\
\hline & Euroline (112) & 14.29 & 68.8 & 99.0 & 91.7 & 95.0 & 94.6 & $0.76(0.57-0.94)$ \\
\hline \multirow[t]{2}{*}{ Dog } & ImmunoCAP (36) & 11.11 & 100.0 & 96.9 & 80.0 & 100.0 & 97.2 & $0.87(0.63-1)$ \\
\hline & Euroline (100) & 5.0 & 60.0 & 100 & 100 & 97.9 & 98.0 & $0.74(0.4-1)$ \\
\hline \multirow[t]{2}{*}{ Egg white } & ImmunoCAP (76) & 47.37 & 61.1 & 90.0 & 84.6 & 72.0 & 76.3 & $0.52(0.33-0.71)$ \\
\hline & Euroline (106) & 44.34 & 63.8 & 91.5 & 85.7 & 76.1 & 79.2 & $0.57(0.41-0.72)$ \\
\hline \multirow[t]{2}{*}{ Egg yolk } & ImmunoCAP (31) & 61.29 & 15.8 & 100.0 & 100.0 & 42.9 & 48.4 & $0.13(-0.02-0.27)$ \\
\hline & Euroline (61) & 49.18 & 33.3 & 93.5 & 83.3 & 59.2 & 63.9 & $0.27(0.08-0.47)$ \\
\hline \multirow[t]{2}{*}{ Cow's milk } & ImmunoCAP (78) & 24.36 & 68.4 & 91.5 & 72.2 & 90.0 & 85.9 & $0.61(0.4-0.82)$ \\
\hline & Euroline (113) & 21.24 & 25 & 93.3 & 50.0 & 82.2 & 78.8 & $0.22(0.01-0.44)$ \\
\hline \multirow[t]{2}{*}{ Wheat } & ImmunoCAP (72) & 22.22 & 43.8 & 92.9 & 63.6 & 85.2 & 81.9 & $0.41(0.15-0.67)$ \\
\hline & Euroline(95) & 20.0 & 26.3 & 97.4 & 71.4 & 84.1 & 83.2 & $0.31(0.07-0.55)$ \\
\hline \multirow[t]{2}{*}{ Shrimp } & ImmunoCAP (72) & 45.83 & 51.5 & 84.6 & 73.9 & 67.3 & 69.4 & $0.37(0.16-0.58)$ \\
\hline & Euroline (154) & 24.03 & 35.1 & 94.0 & 65.0 & 82.1 & 79.9 & $0.35(0.17-0.52)$ \\
\hline \multirow[t]{2}{*}{ Crab } & ImmunoCAP (17) & 35.29 & 66.7 & 81.8 & 66.7 & 81.8 & 76.5 & $0.49(0.05-0.92)$ \\
\hline & Euroline (19) & 36.84 & 71.4 & 91.7 & 83.3 & 84.6 & 84.2 & $0.65(0.29-1)$ \\
\hline
\end{tabular}

Der p, Dermatophagoides pteronyssinus; Der f, Dermatophagoides farinae

$\mathrm{PPV}$, positive predictive value; NPV, negative predictive value

$\mathrm{n}$, number of participants

The value of kappa index was interpreted according to the following scale: $<0$ : poor agreement, $0-0.2$ : slight agreement,0.21-0.40: fair agreement, 0.41-0.60: moderate agreement, 0.61-0.80: substantial agreement, 0.81-1.00: perfect agreement. 
Table 2. The correlaton analysis between sIgE level $(\mathrm{kU} / \mathrm{L})$ measured by ImmunoCAP and Euroline and the result of SPT (diameter of wheal in $\mathrm{mm}$.)

\begin{tabular}{|c|c|c|c|c|c|c|c|c|c|}
\hline \multirow[t]{2}{*}{ Allergen } & \multicolumn{3}{|c|}{ ImmunoCAP and SPT } & \multicolumn{3}{|c|}{ Euroline and SPT } & \multicolumn{3}{|c|}{ ImmunoCAP and Euroline } \\
\hline & Spearman's rho & p-value & $\mathbf{n}$ & Spearman's rho & p-value & $\mathbf{n}$ & Spearman's rho & p-value & $\mathbf{n}$ \\
\hline Der $\mathrm{p}$ & 0.788 & $<0.001$ & 88 & 0.840 & $<0.001$ & 161 & 0.843 & $<0.001$ & 107 \\
\hline Der $\mathrm{f}$ & 0.852 & $<0.001$ & 64 & 0.755 & $<0.001$ & 87 & 0.813 & $<0.001$ & 68 \\
\hline Cat & 0.640 & $<0.001$ & 26 & 0.574 & $<0.001$ & 112 & 0.626 & $<0.001$ & 38 \\
\hline Dog & 0.579 & $<0.001$ & 36 & 0.454 & $<0.001$ & 100 & 0.428 & 0.002 & 52 \\
\hline Egg white & 0.606 & $<0.001$ & 76 & 0.684 & $<0.001$ & 106 & 0.901 & $<0.001$ & 96 \\
\hline Egg yolk & 0.534 & 0.002 & 31 & 0.614 & $<0.001$ & 61 & 0.779 & $<0.001$ & 33 \\
\hline Cow's milk & 0.574 & $<0.001$ & 78 & 0.418 & $<0.001$ & 113 & 0.619 & $<0.001$ & 103 \\
\hline Wheat & 0.390 & 0.001 & 72 & 0.395 & $<0.001$ & 95 & 0.686 & $<0.001$ & 96 \\
\hline Shrimp & 0.623 & $<0.001$ & 72 & 0.664 & $<0.001$ & 154 & 0.707 & $<0.001$ & 101 \\
\hline Crab & 0.573 & 0.016 & 17 & 0.667 & 0.002 & 19 & 0.735 & $<0.001$ & 44 \\
\hline
\end{tabular}

Values presented as correlation. P-value corresponds to Spearman's rho correlation; Der p, Dermatophagoides pteronyssinus; Der $\mathrm{f}$, Dermatophagoides farinae; n, number of participants; NA, not applicable

\section{Correlation between specific IgE measurement using Immuno- CAP and Euroline with SPT}

The correlation of both sIgE assays and SPT was analyzed. For most of the allergens, both Euroline and ImmunoCAP revealed good correlation with SPT $(\mathrm{r}=0.395-0.840, \mathrm{p} \leq 0.002$ and $\mathrm{r}=0.390-0.852, \mathrm{p} \leq 0.016$, respectively). Both sIgE assays have significant correlation for almost all allergens $(\mathrm{r}=0.626$ $0.901, \mathrm{p}<0.001)$ except for dog. For food allergen components, both $\operatorname{sgE}$ assays have good correlation and agreement $(\mathrm{r}=0.816-0.952, \mathrm{p}<0.001$; agreement $=87.0-92.9 \%$, respectively $)$ (Table 2 and 3, Figure 1).
Table 3. Agreement and correlation between Euroline and ImmunoCAP for food components

\begin{tabular}{lcccc}
\hline $\begin{array}{l}\text { Food } \\
\text { component (n) }\end{array}$ & Agreement & $\begin{array}{c}\text { Kappa } \\
(\mathbf{9 5 \% C I})\end{array}$ & $\begin{array}{c}\text { Spearman's } \\
\text { rho }\end{array}$ & p-value \\
\hline $\begin{array}{l}\text { Gluten / } \\
\text { Omega-5 }\end{array}$ & $87.0 \%$ & $\begin{array}{c}0.725 \\
(0.446-1)\end{array}$ & 0.816 & $<0.001$ \\
$\begin{array}{l}\text { Gliadin (23) } \\
\text { Ovalbumin (28) }\end{array}$ & $92.9 \%$ & $\begin{array}{c}0.757 \\
(0.436-1)\end{array}$ & 0.883 & $<0.001$ \\
\hline Ovomucoid (28) & $92.9 \%$ & $\begin{array}{c}0.851 \\
(0.654-1)\end{array}$ & 0.952 & $<0.001$ \\
\hline
\end{tabular}

n, number of participants
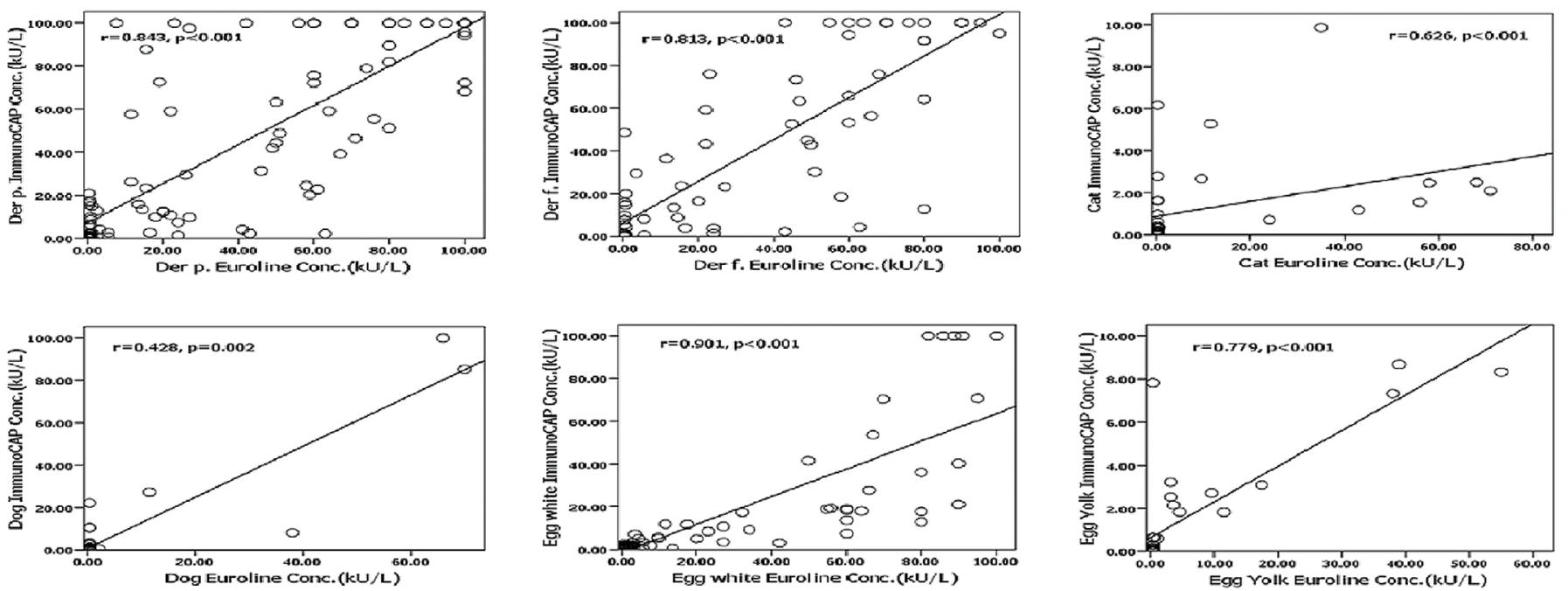

Figure 1. Scatterplots of correlation analysis of sIgE levels $(\mathrm{kU} / \mathrm{L})$ between Euroline and ImmunoCAP for 4 aeroallergens, 6 food allergens and 3 food components. Each dot represents the sIgE of one patient. 

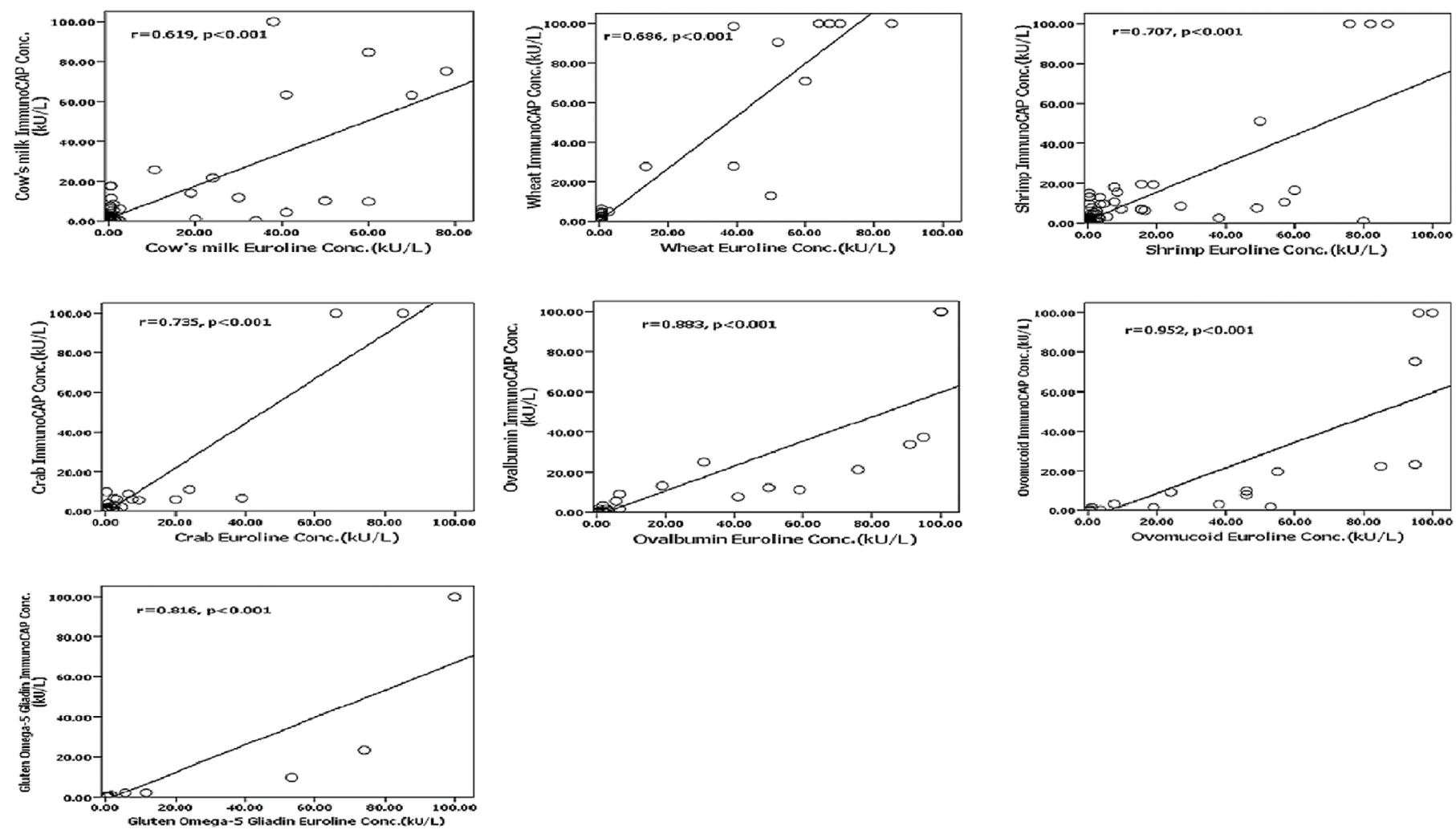

Figure 1. (Continued)

\section{ROC curve analysis of each allergen}

The data of individuals whom had all 3 tests (SPT, Euroline and ImmunoCAP) performed was analyzed by ROC curve (Table 4). The sIgE level measured by Euroline and ImmunoCAP

were plotted against the result of SPT. Area under the curve (AUC), maximum sensitivity, maximum specificity, index $\mathrm{Q}^{*}$ (the maximum value that sensitivity and specificity can be

Table 4. ROC curve analysis for ImmunoCAP and Euroline base on the result of SPT

\begin{tabular}{|c|c|c|c|c|c|}
\hline Allergen (n) & System & $\operatorname{AUC}(95 \% \mathrm{CI})$ & p-value & AccuMax $^{\psi}$ & Cutoff valve $(\mathrm{kU} / \mathrm{L})^{\theta}$ \\
\hline \multirow{2}{*}{ Der p (79) } & ImmunoCAP & $0.996(0.986-1.005)$ & $<0.001^{*}$ & 97.5 & 0.39 \\
\hline & Euroline & $0.933(0.875-0.990)$ & $<0.001^{*}$ & 89.9 & 0.41 \\
\hline \multirow[t]{2}{*}{$\operatorname{Der} \mathrm{f}(52)$} & ImmunoCAP & 0.996(0.984-1.008) & $<0.001^{*}$ & 98.1 & 0.88 \\
\hline & Euroline & $0.929(0.860-0.998)$ & $<0.001^{*}$ & 90.4 & 2.1 \\
\hline \multirow[t]{2}{*}{ Cat (26) } & ImmunoCAP & $0.745(0.547-0.943)$ & $0.036^{*}$ & 76.9 & 0.655 \\
\hline & Euroline & $0.767(0.583-0.950)$ & $0.022^{\star}$ & 73.1 & 0.525 \\
\hline \multirow[t]{2}{*}{$\operatorname{Dog}(36)$} & ImmunoCAP & $0.978(0.929-1.026)$ & $<0.001^{*}$ & 97.2 & 5.69 \\
\hline & Euroline & $0.750(0.484-1.016)$ & 0.056 & 91.7 & 5.925 \\
\hline \multirow[t]{2}{*}{ Egg white (74) } & ImmunoCAP & $0.839(0.748-0.929)$ & $<0.001^{*}$ & 79.7 & 1.18 \\
\hline & Euroline & $0.862(0.779-0.945)$ & $<0.001^{*}$ & 79.7 & 1.6 \\
\hline \multirow[t]{2}{*}{ Egg yolk (31) } & ImmunoCAP & $0.834(0.693-0.976)$ & $0.005^{*}$ & 80.6 & 0.07 \\
\hline & Euroline & $0.731(0.556-0.906)$ & 0.055 & 64.5 & 0.475 \\
\hline \multirow[t]{2}{*}{ Cow's milk (76) } & ImmunoCAP & $0.804(0.701-0.907)$ & $<0.001^{*}$ & 81.6 & 2.34 \\
\hline & Euroline & $0.700(0.575-0.826)$ & $0.003^{*}$ & 72.4 & 0.355 \\
\hline \multirow[t]{2}{*}{ Wheat (69) } & ImmunoCAP & $0.638(0.500-0.776)$ & 0.051 & 68.1 & 1.395 \\
\hline & Euroline & $0.618(0.481-0.754)$ & 0.096 & 65.2 & 0.355 \\
\hline \multirow[t]{2}{*}{ Shrimp (69) } & ImmunoCAP & $0.834(0.736-0.932)$ & $<0.001^{*}$ & 81.2 & 0.605 \\
\hline & Euroline & $0.727(0.605-0.849)$ & $0.002^{*}$ & 69.6 & 0.585 \\
\hline \multirow[t]{2}{*}{ Crab (17) } & ImmunoCAP & $0.889(0.729-1.049)$ & $0.007^{*}$ & 82.4 & 2.485 \\
\hline & Euroline & $0.875(0.691-1.059)$ & $0.009^{*}$ & 88.2 & 1.5 \\
\hline
\end{tabular}


mutual achieved) and the cutoff value were analyzed. Both Euroline and ImmunoCAP revealed comparable AUC. ImmunoCAP revealed slightly higher accuracy for most allergens except cat and egg white. Although, there were some differences of $\mathrm{Q}^{*}$ cutoff value between the 2 systems, most of cutoff values of each allergen of the 2 systems were at the same class.

\section{Discussion}

The diagnosis of IgE-mediated allergy is based on clinical symptoms and the evidence of sensitization. SPT is the primary tool for allergist in detection of the causative allergen. It is convenient, rapid, no machine requirement and inexpensive. However, the result of SPT may depend on the performers and can also be affected by medications while in vitro $\operatorname{sigE}$ determination can overcome the disadvantage of SPT.

Currently, a number of in vitro sIgE determination systems are available. Each of them uses different allergen sources and distinct $\operatorname{IgE}$ detection system. Thus, the sIgE results measured by one system might not be interchangeable with the others. ${ }^{8}$ Our study evaluated serum sIgE level of common allergens measured by Euroline (Immunoblotting system) and ImmunoCAP (Fluorescence enzyme immune assay) in comparision with SPT.

When compared the two SIgE determination systems to SPT, our results revealed that both Euroline and ImmunoCAP provided good to fair performance depending on types of allergens. For house dust mites (Der $p$ and Der f), the excellent concordance has been identified between SPT and both sIgE determination systems. For other allergens except crab, Euroline performed with lower sensitivity but higher specificity compared to ImmunoCAP. Euroline also provided slightly higher agreement with SPT than ImmunoCAP for egg white and crab allergens. The discordance between sIgE and SPT demonstrated in our study is in line with previous reports demonstrating some discordance between sIgE and SPT. The discordance rates varied depending on type of allergens and patients' factors. ${ }^{7,11,12}$ Chauveau et al demonstrated the good agreement between SPT and sIgE measured with Allergy Screen Test Panel (Mediwiss Analytic, Moers, Germany) for house dust mites and a poor agreement for cat, dog, alternaria, and grass pollen. ${ }^{13}$ de Vos et al studied the concordance between SPT for 7 common aeroallergens (grass pollen, ragweed pollen, dust mite, cockroach, mouse, cat, and dog) and sIgE testing using Immulite 2000 3gAllergy $^{\mathrm{T}}$ system (Siemens AG, Munich, Germany) in 40 atopic inner-city children aged 18 to 48 months. The study revealed a fair correlation for most allergens and no correlation between SPT grade and sIgE level for dog. ${ }^{14}$ The discrepancies of the result of SPT and sIgE assays in our study can be explained by the differences in composition of allergens used in SPT and sIgE assays as well as patients' factors.

When the two sIgE determination systems were compared, there was a significant correlation of the sIgE levels for most allergens. The correlation was strong for Der $\mathrm{p}$, Der $\mathrm{f}$ and egg white but weak for dog. Interestingly, both Euroline and ImmunoCAP have good concordance in detection of sIgE for food allergen components.
When compared Euroline with ImmunoCAP by ROC analysis, there were some marginal discrepancies between both systems. ImmunoCAP revealed slightly higher accuracy in detection of SIgE to majority of allergens used in this study except cat and egg white. The discrepancies between both systems could be due to the differences in assay technique as well as the composition and concentration of allergens.

It is important to emphasize that a positive serum $\operatorname{sgE}$ or SPT indicates a sensitization to an allergen and is not equivalent to a clinical diagnosis. This limitation highlights the need for the clinician to use medical history together with knowledge of the test characteristics to select and interpret tests properly.

To the best of our knowledge, our study has been the first to evaluate performance of Euroline, the system using immunoblotting technique and another system using fluorescence enzyme-immunoassay (ImmunoCAP) with SPT. Based on SPT, Euroline shows comparable performance with ImmunoCAP for several common allergens. Furthermore, the levels of sIgE detected by Euroline significantly correlated with those measured by ImmunoCAP. Interestingly, there was good concordance between the two systems in detecting sIgE for food allergen components. Therefore, our data suggested that Euroline provided high accuracy for several common allergens and food components. Since system of Euroline has some advantages as prior mentioned, further study with more allergens and the correlation with clinical data should be performed.

\section{Acknowledgement}

This study was supported by the $90^{\text {th }}$ Year Anniversary Ratchadapiseksompotch Endowment Fund, Faculty of Medicine, Chulalongkorn University, Thailand (RA56/008).

\section{References}

1. Cox L, Williams B, Sicherer S, Oppenheimer J, Sher L, Hamilton R, et al. Pearls and pitfalls of allergy diagnostic testing: report from the American College of Allergy, Asthma and Immunology/American Academy of Allergy, Asthma and Immunology Specific IgE Test Task Force. Ann Allergy Asthma Immunol. 2008;101(6):580-92.

2. van der Zee JS, de Groot H, van Swieten P, Jansen HM, Aalberse RC. Discrepancies between the skin test and IgE antibody assays: study of histamine release, complement activation in vitro, and occurrence of allergen-specific IgG. J Allergy Clin Immunol. 1988;82(2):270-81.

3. Sicherer SH, Wood RA, American Academy of Pediatrics Section On A, Immunology. Allergy testing in childhood: using allergen-specific IgE tests. Pediatrics. 2012;129(1):193-7.

4. Wood RA, Phipatanakul W, Hamilton RG, Eggleston PA. A comparison of skin prick tests, intradermal skin tests, and RASTs in the diagnosis of cat allergy. J Allergy Clin Immunol. 1999;103(5 Pt 1):773-9.

5. Crobach MJ, Hermans J, Kaptein AA, Ridderikhoff J, Petri H, Mulder JD. The diagnosis of allergic rhinitis: how to combine the medical history with the results of radioallergosorbent tests and skin prick tests. Scand J Prim Health Care. 1998;16(1):30-6.

6. Gendo K, Larson EB. Evidence-based diagnostic strategies for evaluating suspected allergic rhinitis. Ann Intern Med. 2004;140(4):278-89.

7. de Vos G, Nazari R, Ferastraoaru D, Parikh P, Geliebter R, Pichardo Y, et al. Discordance between aeroallergen specific serum IgE and skin testing in children younger than 4 years. Ann Allergy Asthma Immunol. 2013; 110(6):438-43.

8. Wood RA, Segall N, Ahlstedt S, Williams PB. Accuracy of IgE antibody laboratory results. Ann Allergy Asthma Immunol. 2007;99(1):34-41.

9. Lee YW, Sohn JH, Lee JH, Hong CS, Park JW. Allergen-specific IgE measurement with the IMMULITE 2000 system: intermethod comparison of detection performance for allergen-specific IgE antibodies from Korean allergic patients. Clin Chim Acta. 2009;401(1-2):25-32. 
10. Ricci G, Capelli M, Miniero R, Menna G, Zannarini L, Dillon P, et al. A comparison of different allergometric tests, skin prick test, Pharmacia UniCAP and ADVIA Centaur, for diagnosis of allergic diseases in children. Allergy. 2003;58(1):38-45.

11. Calabria CW, Dietrich J, Hagan L. Comparison of serum-specific IgE (ImmunoCAP) and skin-prick test results for 53 inhalant allergens in patients with chronic rhinitis. Allergy Asthma Proc. 2009;30(4):386-96.

12. Cho JH, Suh JD, Kim JK, Hong SC, Park IH, Lee HM. Correlation between skin-prick testing, individual specific IgE tests, and a multiallergen IgE assay for allergy detection in patients with chronic rhinitis. Am J Rhinol Allergy. 2014;28(5):388-91.
13. Chauveau A, Dalphin ML, Mauny F, Kaulek V, Schmausser-Hechfellner E, Renz H, et al. Skin prick tests and specific IgE in 10-year-old children: Agreement and association with allergic diseases. Allergy. 2017:1-9.

14. De Vos G, Nazari R, Ferastraoaru D, Parikh P, Geliebter R, Pichardo Y, et al. Discordance between aeroallergen specific serum IgE and skin testing in children younger than 4 years. Ann Allergy Asthma Immunol. 2013 Jun 30;110(6):438-43. 